\title{
ROBUST ESTIMATION OF FIELD POTENTIAL DURATION IN MULTI-ELECTRODE ARRAY SIGNALS \\ L. BATISTA ${ }^{1}$, T. BASTOGNE $1,2,3$ \\ ${ }^{1}$ CYBERnano, ${ }^{2}$ CRAN UL-CNRS UMR 7039, 2INRIA BIGS
}

Background. Electrical signals measured by microelectrode arrays (MEAs) allow non-invasive long-term monitoring of cardiomyocyte cultures. However, variability of local responses between electrodes and noise makes difficult the accurate estimation of the field potential duration (FPD). False peaks are often detected and falsify the FDP estimated value, which finally requires for the user to perform some manual corrections for each well. This drawback not only slows down the analysis but also introduces arbitrary estimations and inter-individual errors.

\section{Objectives}

The main objective is to develop a new estimation method of FPD taking advantages of all the information contained in the signals measured by all the electrodes of the same well. The motivations are to automatically compute FPD and to improve the accuracy and robustness of the estimation compared to existing techniques already implemented in the available technologies.

\section{Methods}

The new estimation method relies on a Monte Carlo technique, i.e. a random sampling simulation process adapted to MEA signals. To test it, we used a MEA data set measured by an Axion Maestro platform with $\mathrm{N}=8$ electrodes in each well. For the validation step, the new FPD estimates were compared to two sets of estimated values. The first set is provided by an expert who observed and manually estimate the FPD value for each sweep and each well. The second set of estimates is automatically computed by the platform software. Comparisons were repeated on 128 items (64 wells at two different time instants, before and after compound administration). Histograms and correlation coefficients of the estimation errors were compared for the three estimation techniques. Statistical analysis was carried out in the R computing environment.

\section{Results}

The correlation coefficient between the expert FPD values and the FPD estimates given by the two tested estimation techniques has been drastically improved with the new statistical method developed in this study (before: $r=0.14$ vs $r=0.44$ with the new method). By removing 5 outliers among the 128 estimates, we obtained a correlation coefficient: $r=0.95$, which is fully acceptable.

\section{Conclusion}

This study demonstrates the practical efficacy of a new estimation technique to automatically estimate the FPD parameter from MEA data.
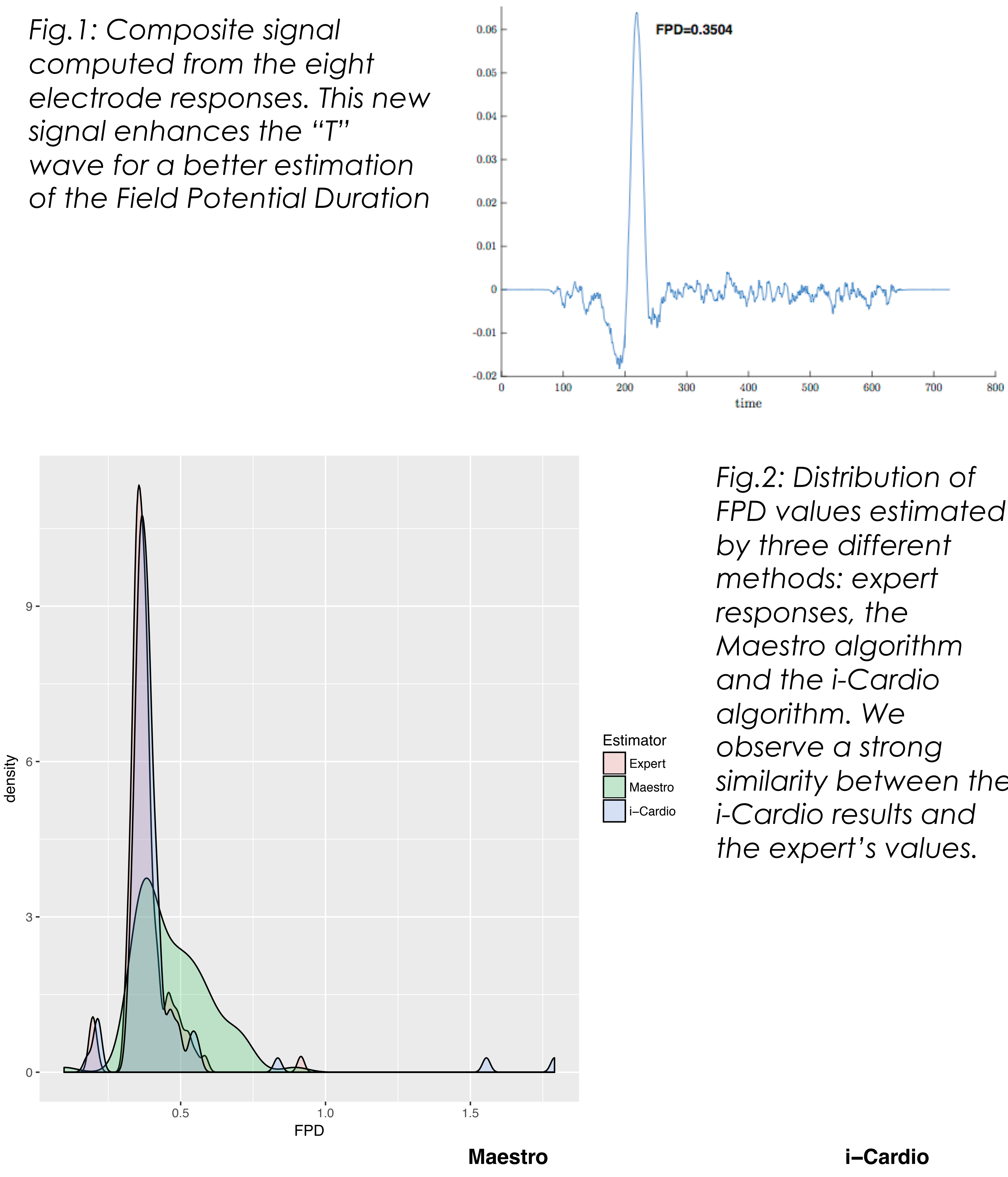

Fig.2: Distribution of FPD values estimated by three different methods: expert responses, the Maestro algorithm and the i-Cardio algorithm. We observe a strong similarity between the $i$-Cardio results and the expert's values.

Fig.3: Results of the FPD estimations from MEA signals. Comparison of correlations between the two estimation methods and the expert values (left: $r=0.14$ right: r:0.44)
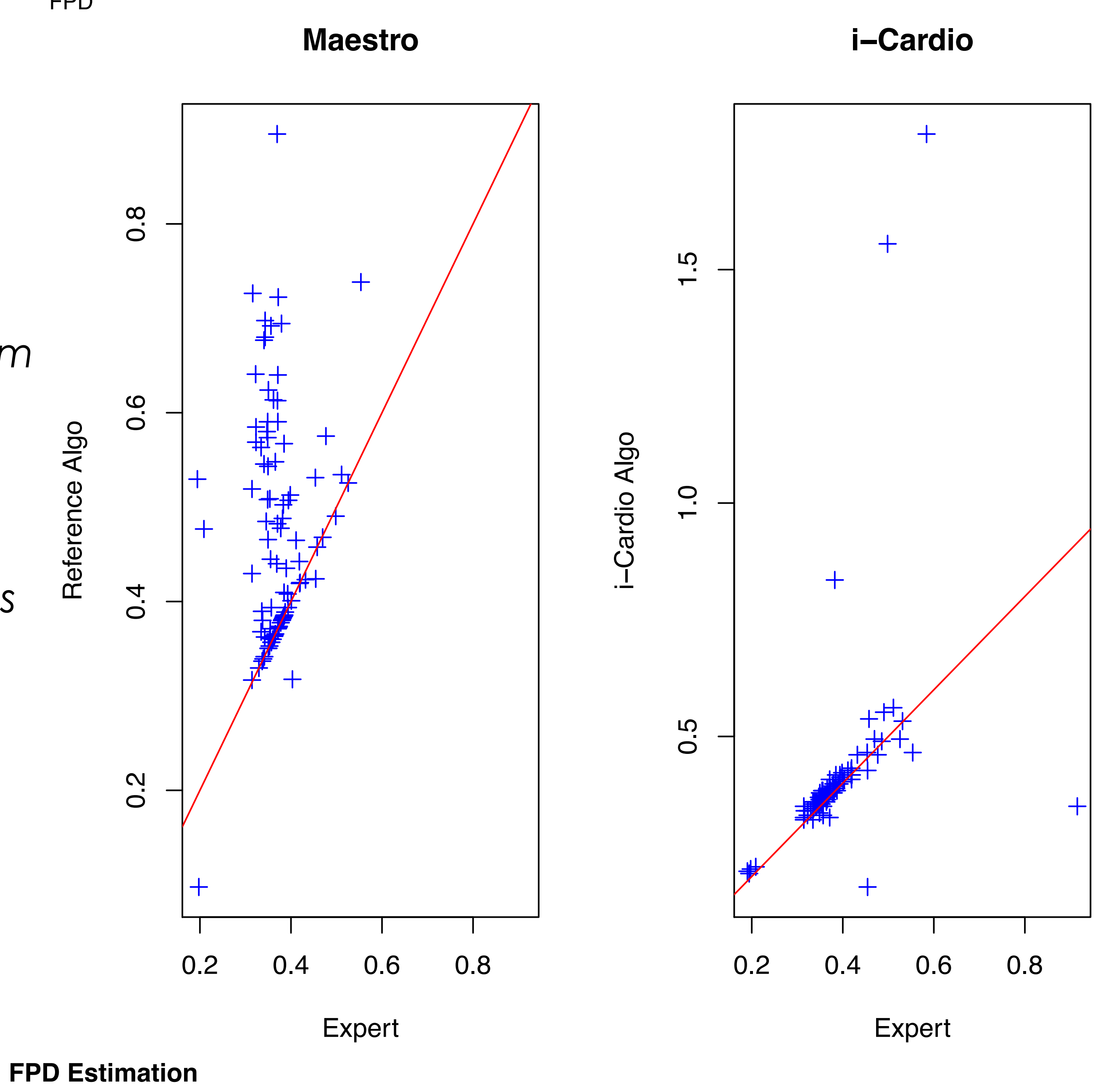

Fig.4: Comparison of

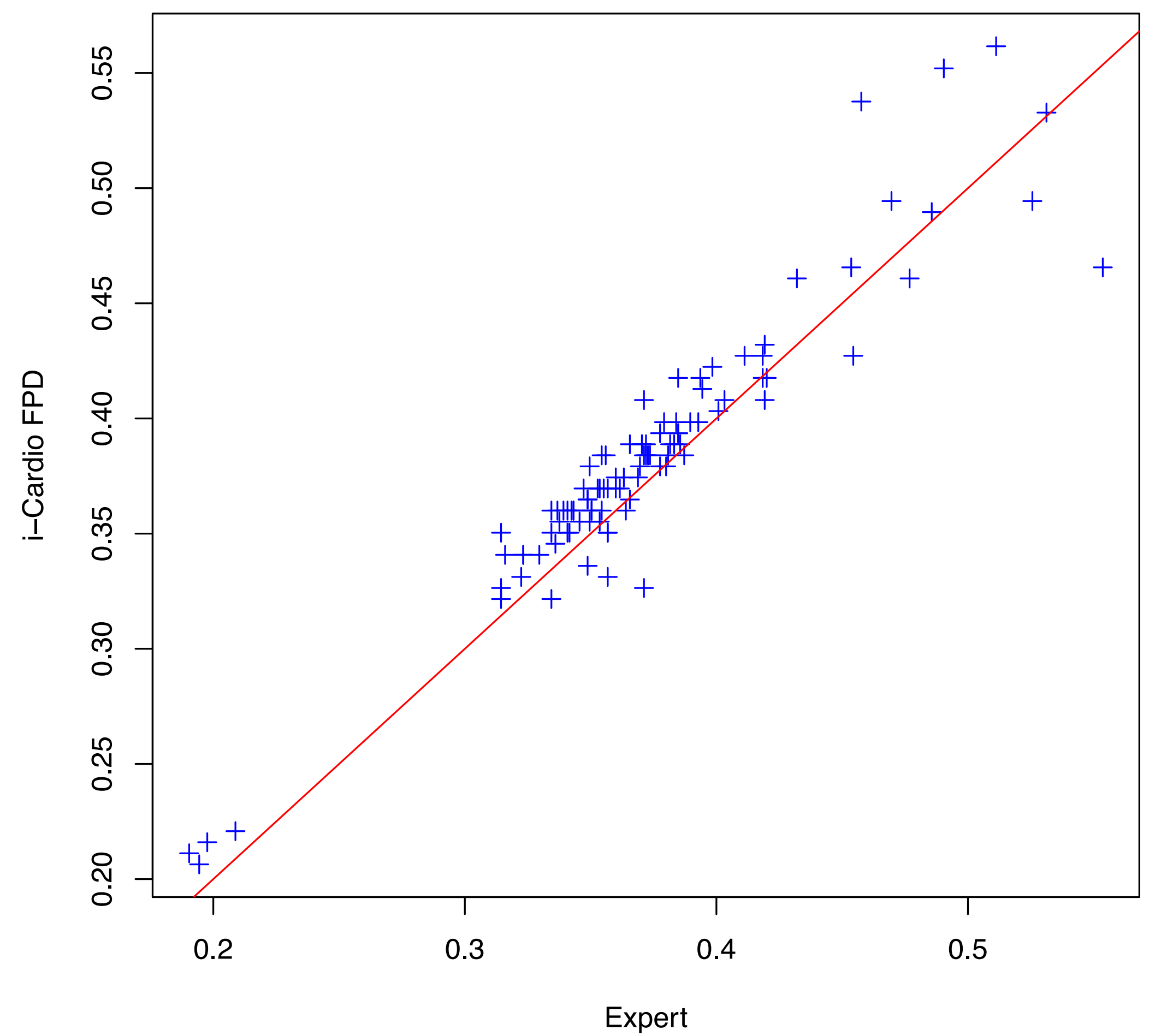
correlations between the iCardio estimation method and the expert values after elimination of five estimation. Correlation coeff: $r=0.95$ 